Review Article

\title{
Does internal cardiac massage play a role in ROSC after prolonged CPR ? : Our experience in a tertiary care hospital
}

\author{
Manjunath R. Kamath ${ }^{1}$, Krishna Prasad P. ${ }^{2}$, M. Gopalakrishnan ${ }^{3}$ \& Amith Kiran ${ }^{4}$ \\ ${ }^{1,2}$ Associate Professors, Department of Anaesthesiology \& Critical Care, ${ }^{3,4}$ Associate Professor, Department of \\ Cardiothoracic Surgery, K.S. Hegde M edical Academy, Nitte University, M angalore, Karnataka, India.
}

Correspondence

Manjunath R. Kamath

Associate Professor, Department of Anaesthesiology \& Critical Care, K.S. Hegde M edical Academy, Deralakatte, M angalore - 575018, Karnataka. E-mail : manjunathrkamath@gmail.com

\begin{abstract}
Cardiopulmonary resuscitation (CPR) is a life-saving skill involving chest compressions \& ventilation to pump oxygenated blood through the vital organs of the body. Among different techniques and devices for chest compression, no single method has been definitively shown to produce the best outcome and hence, direct comparisons between them are not possible. Internal cardiac massage is the manual squeezing of the heart through a surgical incision into the chest cavity, when the chest is already open for cardiac surgery. Unlike the usual cardiac arrest scenarios, internal cardiac massage is employed mostly in cardiothoracic surgical patients and will have more chance for survival and favorable neurologic outcomes across all durations of CPR. We describe two cases of return of spontaneous circulation (ROSC) after prolonged CPR with internal cardiac massage performed in hospital setting.
\end{abstract}

Keywords: Cardiopulmonary resuscitation (CPR); in-hospital cardiac arrest (IHCA); internal cardiac massage; return of spontaneous circulation (ROSC)

\section{Introduction}

Cardiopulmonary resuscitation (CPR) is an emergency procedure for manually preserving brain functions by spontaneous circulation and breathing in a person who has suffered cardiac arrest. Though CPR alone is unlikely to restart the heart as it only restores partial flow of oxygenated blood to the brain and heart. It delays tissue death and extends the brief "window of opportunity" for a successful resuscitation without permanent brain damage. ${ }^{1}$

If hemodynamics, neurological recovery and overall survival do not improve with optimal external chest compressions, consideration should be given to convert to open-chest cardiac massage whenever prolonged resuscitation is expected and wherever feasible. Open massage produces superior cardiac output, better perfusion pressure, improved neurological outcome and

\begin{tabular}{|c|}
\hline Access this article online \\
\hline Quick Response Code \\
\hline
\end{tabular}

increased likelihood of successful resuscitation. $2,3,4,56$

Data suggest that converting to open cardiac massage after 20 minutes of closed chest CPR does not improve survival. ${ }^{7}$
When cardiac arrest occurs inside the hospital, outcome is better because CPR and internal cardiac massage can be started early.

\section{Material and M ethods}

Patient 1

A patient aged 58 years who presented with history of exertional dyspnea was evaluated and diagnosed to have triple vessel disease with $80 \%$ lesion in the mid left anterior descending artery (LAD) , first diagonal (D1) 70\% lesion, first obtuse marginal (OM 1) 70\% lesion and right coronary artery (RCA) $100 \%$ lesion. All the pre-operative laboratory investigations were within normal limits. Echocardiogram (ECHO) revealed dilated left ventricle, ejection fraction $50 \%$, mild hypokinesia of inter-ventricular septum, inferior and posterior wall, severe mitral regurgitation, trivial aortic regurgitation \& trivial tricuspid regurgitation. The patient was on regular medications with beta blockers and sorbitrate. He was scheduled to undergo off-pump coronary artery bypass (OPCAB) and mitral valve replacement under cardiopulmonary bypass (CPB).

After placement of arterial and central venous, lines anaesthesia was induced with Inj.fentanyl 250microgram, Inj. midazolam $5 \mathrm{mg} \&$ Inj. propofol 50mg. Trachea was intubated with no. $8.5 \mathrm{~mm}$ cuffed endotracheal tube using vecuronium $6 \mathrm{mg}$ as muscle relaxant. 
Midline sternotomy was done. After harvesting internal mammary artery (LIMA) \& saphenous vein (GSV) and heparinisation, LIMA was anastomosed to LAD off-pump. CPB was initiated with routine aortic \& bi-caval cannulations, cooled to $28^{\circ} \mathrm{C}$ and cardioplegia administered. GSV was grafted to OM1, mitral valve replaced with $29 \mathrm{~mm}$ TTK-CHITRA mechanical tilting-disc type valve. CPB discontinued after fulfilling all criteria. Proximal grafting to aorta was completed, heparin reversed with protamine, hemostasis achieved $\&$ chest closed.

Inotropes on flow were Inj. adrenaline $0.05 \mathrm{mcg} / \mathrm{kg}$ and Inj. dobutamine $5 \mathrm{mcg} / \mathrm{kg}$. Patient was shifted to the postoperative ward \& electively ventilated. Hemodynamics and blood gases remained stable.

Three hours post-operatively, blood pressures (BP) started gradually reducing from $100 / 70 \mathrm{~mm} \mathrm{Hg}$ to $60 / 40 \mathrm{~mm} \mathrm{Hg}$ and heart rate (HR) dropped from 70 to $40 \mathrm{bpm}$. Treatment was attempted with increased infusion rate of inotropes, pacing and Inj. atropine $0.6 \mathrm{mg}$ intravenous. In spite of all these measures, the patient developed cardiac arrest.

CPR was immediately commenced according to the American Heart Association (AHA) guidelines. In view of the possibility of surgical related complication, chest was opened bedside and internal cardiac massage instituted. Medications like Inj. adrenaline (1mg every 3minutes) totally $4 \mathrm{mg}$, Inj. calcium gluconate $(10 \mathrm{ml}$ of $10 \%$ solution to correct hyperkalemia) and Inj. sodium bicarbonate (to correct acidosis) according to the base deficit were administered intravenously. In addition, Inj. heparin $100 \mathrm{mg}$ was administered to treat coronary vessel thrombosis if any. Simultaneously causes for the asystole and subsequent cardiac arrest were sought for. Kinking of the grafts was ruled out. Ventricular fibrillation was treated with internal defibrillation along with Inj. lignocaine $100 \mathrm{mg}$ \& subsequently with Inj. amio darone $250 \mathrm{mg}$.

Patient was successfully resuscitated after one hour of continuous internal cardiac massage as indicated by ROSC, stabilization of blood pressures at $110 / 70 \mathrm{mmHg} \&$ pulse of
$100 \mathrm{bpm}$. Inotropes on-flow at that time were adrenaline $0.05 \mathrm{mcg} / \mathrm{kg} / \mathrm{min}$, dopamine $10 \mathrm{mcg} / \mathrm{kg} / \mathrm{min}$ and dobutamine $5 \mathrm{mcg} / \mathrm{kg} / \mathrm{min}$. Hyperglycemia was controlled with insulin infusion. Patient remained stable overnight. Patient was sedated, paralyzed \& electively ventilated.

Patient developed fever on post-operative day (POD) 1 evening. Antibiotics were stepped up. Patient was further sedated \& ventilated for 2 days maintaining stable hemodynamics. After full neurological recovery and meeting all extubation criteria, patient was extubated on POD2 evening. Vitals remained stable, inotropes were slowly tapered \& drains removed on POD3. Invasive lines \& pacing wires were removed on POD4 and patient was shifted to the ward. Subsequently he had an uneventful post-operative period till discharge. The cause of cardiac arrest remained unknown, though a mechanical valve dysfunction wassuspected.

\section{Patient 2}

A 3 year old girl weighing $10 \mathrm{~kg} \&$ post-operative case of surgical atrial septal defect (ASD) closure 11/2 months back, presented to our center with complains of on and off fever, loss of appetite and purulent discharge from the sternal wound area since 1 month.

On examination, she was febrile. Local examination revealed infected old median sternotomy wound and exposed sternal wires, eroded manubrio-sternal junction and blood clots. Diagnosis of surgical site infection with a possibility of pseudo-aneurysm of aorta at the old aortic cannulation site was arrived at.

Laboratory reports revealed hemoglobin $(\mathrm{Hb})$ of $7.3 \mathrm{~g} \%$, raised white blood cell counts. Chest X-Ray revealed mild pleural effusion \& ECHO showed mild pericardial effusion. She was posted for emergency re-exploration of the chest $\&$ wound debridement with adequate blood products available.

Patient was shifted to the operation theatre; standard American Society of Anaesthesiologists (ASA) monitors (Electrocardiogram, non-invasive BP, pulse oximetry) were connected. Adequate venous access was secured. Arterial 
$\&$ central venous lines inserted. Airway was secured after rapid sequence induction with Inj. propofol $20 \mathrm{mg} \&$ Inj. succinylcholine 20mg. Patient was intubated with $6 \mathrm{~mm}$ cuffed oral endotracheal tube (COETT). Anaesthesia was maintained with $\mathrm{N}_{2} \mathrm{O} \& \mathrm{O}_{2}\left(\mathrm{FiO}_{2} \mathrm{0.5}\right)$ and $1 \mathrm{MAC}$ isoflurane with Inj. atracurium boluses.

Old sternotomy incision was re-explored. Sternal wires were removed and thorough wound debridement was performed. Chest was closed after hemostasis.

After reversing the residual neuromuscular block, patient was extubated. At the time of shifting the patient out of operation room (OR), alarming bleeding was noticed from the sternal wound site. She was immediately shifted back to the $\mathrm{OR}$ and re-exploration was decided.

Adequate units of fresh, compatible whole blood were requisitioned for. Then she was administered general anaesthesia in the same sequence as described above.

On re-exploration, profuse bleeding from surgical site was noticed. There was an immediate fall in blood pressures to $30 \mathrm{~mm} \mathrm{Hg}$ systolic after re-opening the chest. 1 unit of whole blood was transfused. Bleeding point at the old aortic cannulation site was identified and controlled simultaneously. A rent measuring about $2 \mathrm{~cm}$ over the old aortic cannulation site on the ascending aorta was sutured in 2 layers. Blood pressures were sustained with blood transfusion \& phenylephrine boluses of $20 \mathrm{mcg}$.

Patient fibrillated \& developed cardiac arrest after 5 minutes of initiation of surgery. She was ventilated with $100 \%$ oxygen; CPR was initiated with internal cardiac massage. Defibrillation was attempted with 5 J shock increasing in strength up to 20J after every 3 minutes of internal cardiac massage. Patient was administered Inj. adrenaline ( $0.1 \mathrm{mg} / \mathrm{kg}$ of 1:10000 dilution) every 3 minutes according to the AHA CPR protocols. Cardiac massage was continued. Inj. xylocard 100mg and Inj. amio darone $250 \mathrm{mg}$ bolus in 20 minutes were administered. Cerebral protection was achieved with Inj. thiopentone $50 \mathrm{mg}$ bolus $\&$ ice packs. Various causes of ventricular fibrillation were considered. CPR was continued for 45 minutes without interruptions.

At the $46^{\text {th }}$ minute, cardiac activity was noticed $\&$ heart maintained normal sinus rhythm with good ejections. All the bleeding points were sought for thoroughly and hemostasis achieved. Sternum was subsequently closed.

Stable hemodynamics was achieved with inotropes infusions of adrenaline $0.05 \mathrm{mcg} / \mathrm{kg} / \mathrm{min}$ and dobutamine $5 \mathrm{mcg} / \mathrm{kg} / \mathrm{min}$. Patient was shifted to the postoperative ward, electively ventilated overnight and was extubated next day after fulfilling standard extubation criteria \& normal blood gases.

\section{Results}

In both these scenarios, patients survived because of early recognition of the adverse cardiac events, early CPR and prompt institution of internal cardiac massage. Patients did not show any neurological deficits and were haemodynamically stable post resuscitation and at discharge. This was possible due to the occurrence of cardiac arrest in the hospital setting \& specifically so, in the cardiac Operation Room (OR) and cardiac ICU. These areas have advanced monitoring facilities were well equipped \& adequately staffed to handle such emergencies. Rapid institution of internal cardiac massage $\&$ prolonged CPR duration contributed to the return of cardiac activity.

\section{Discussion}

Cardiac arrest is multi-factorial in aetiology and the outcome depends on timely and appropriate interventions. CPR is used on patients in cardiac arrest in order to oxygenate blood and maintain a cardiac output so that vital organs survive. The brain may sustain damage without oxygenated blood for four minutes and suffers irreversible damage after about seven minutes. ${ }^{1}$ Therefore, CPR is effective only if performed within seven minutes of the cessation of blood flow.

Chest compressions should be immediately initiated in a pulseless patient. With a straight downward thrust, the sternum is depressed $1 \frac{1}{2-2} 2$ inches $(4-5 \mathrm{~cm})$ in adults, $1-1 \frac{1}{2}$ inches $(2-4 \mathrm{~cm})$ in children, and then allowed complete recoil. For an infant, compression depth is $1 / 2-1$ inches 
$\left(1 \frac{1}{2}-2^{1 / 2} \mathrm{~cm}\right)$. Compression and release times should be equal.

Chest compressions force blood to flow either by increasing intra-thoracic pressure (thoracic pump) or by directly compressing the heart (cardiac pump). During CPR of short duration, blood flow is created more by the cardiac pump mechanism; as CPR continues, the heart becomes less compliant and the thoracic pump mechanism becomes more important.

In-hospital cardiac arrest (IHCA) has not received the same level of focused research as out of hospital cardiac arrest $(\mathrm{OHCA}) .{ }^{8,9}$ The chances of survival were better when cardiac arrests occurred in the well monitored areas like OR, ICU and cath suites. ${ }^{10}$ This may be due to immediate resuscitation and internal cardiac massage possible in the cardiac ORs.

Yanagidani T et al took a total of 100 minutes to successfully resuscitate a patient without any resulting neurological damage. This was attributed to appropriate open chest massage. They opined that open chest massage should be considered if a case of cardiac arrest due to any coronary artery disease does not respond to the usual cardiopulmonary resuscitation. ${ }^{11}$

Darragh Twomey et al in their study concluded that internal massage was superior to external massage for patients suffering a cardiac arrest after cardiac surgery. ${ }^{12}$ They have shown that closed chest massage generates a cardiac index of around $0.6 \mathrm{l} / \mathrm{min} / \mathrm{m}^{2}$ which rises to $1.3 \mathrm{l} / \mathrm{min} / \mathrm{m}^{2}$ or more with open-chest-CPR, accompanied by even bigger improvements in coronary perfusion pressure. Based on International Liaison Committee on Resuscitation (ILCOR) guidelines, they recommend early conversion to openchest-cardiac massage in such patients in order to significantly improve the quality of cardiopulmonary resuscitation.

Animal studies done by Sanders et al showed that openchest-CPR resulted in $4 / 5$ dog survivors compared to none in their closed-chest group. ${ }^{13}$ Similarly, Kern et al in their study on 29 dogs examined whether open-chest cardiac massage could improve 7 day survival and neurologic function when instituted after the failure of standard closed-chest compression CPR. There was more success in dogs receiving open-chest cardiac massage, (14/14 vs 5/14; p less than .005), $24 \mathrm{hr}$. survival (12/14 vs 4/14; p less than .005), and 7 day survival (11/14 vs $4 / 14$; $p$ less than .02) than in those receiving continued closed-chest compressions. They concluded that resuscitation significantly improved if chest opening is instituted sooner. ${ }^{14}$

Fialka et al in their study observed that patients with blunt trunk trauma and cardiac arrest after hemorrhagic shock may benefit from open-chest CPR. They stated that internal cardiac massage is very effective if started at least within 20 minutes after initiation of traditional CPR methods. ${ }^{15}$

In the modern era, however, thoracotomy and open-chest cardiac massage are not part of routine CPR because of the high incidence of severe complications. Nonetheless, these invasive techniques can be helpful in specific lifethreatening clinical situations that preclude effective closed-chest massage. Possible indications include cardiac arrest associated with penetrating or blunt chest trauma, penetrating abdominal trauma, severe chest deformity, pericardial tamponade and post cardiac surgical patients.

Lim GB in his study observed that many hospitalized children survive after prolonged resuscitation (more than 35 minutes) for cardiac arrest. Over half of survivors of prolonged resuscitation had no neurological sequelae. ${ }^{16}$ Longer duration of CPR may save the lives of children who otherwise would have died.

The 2010 American Heart Association Guidelines for Cardiopulmonary Resuscitation and Emergency Cardiovascular Care acknowledge that there is not yet enough scientific evidence to guide when further efforts of CPR would be futile for an individual patient and hence do not currently recommend a specific duration for resuscitation attempt. ${ }^{17}$

Goldberger et al in their observational study evaluated a 
potential association between duration of CPR and survival after in-hospital cardiac arrest. The evidence remains insufficient to recommend a minimum duration for an inhospital resuscitation attempt. Instead, the duration of CPR should be determined on a case-by-case basis, delivery of higher-quality CPR and coordinated \& fruitful teamwork. ${ }^{18}$

\section{Conclusion}

As we strive \& struggle to realize the goal of full neurological recovery after cardiac arrest, future studies

\section{References}

1. Field JM, Hazinski MF, Sayre MR, Chameides L, Schexnayder SM, Hemphill R, et al. Part 1: Executive summary: 2010 American Heart Association Guidelines for Cardiopulmonary Resuscitation and Emergency Cardiovascular Care. Circulation 2010; 122(18):S640-56.

2. Sanders AB, Kern KB, Atlas M, Bragg S, Ewy GA. Importance of the duration of inadequate coronary perfusion pressure on resuscitation from cardiac arrest. J Am Coll Cardiol. 1985; 6(1):113-118.

3. Stephenson HE Jr, Reid LC, Hinton JW. Some common denominators in 1200 cases of cardiac arrest. Ann Surg. 1953; 137(5):731-744.

4. Weiser FM, Adler LN, Kuhn LA. Hemodynamic effects of closed and open-chest cardiac resuscitation in normal dogs and those with acute myocardial infarction. Am J Cardiol. 1962; 10:555-561.

5. Del Guercio LRM, Feins NR, Cohn JD, Coomaraswamy RP, Wollman SB, State D. Comparison of blood flow during external and internal cardiac massage in man. Circulation 1965; 31 (4S1):171-80.

6. Bircher NG, Safar P. Cerebral preservation during cardiopulmonary resuscitation. Crit Care Med 1985; 13(3):185-90.

7. Geehr EC, Lewis FR, Auerbach PS. Failure of open-heart massage to improve survival after prehospital nontraumatic cardiac arrest. N Engl J Med 1986; 314:1189-1190.

8. Morrison LJ, Neumar RW, Zimmerman JL, Link MS, Newby LK, McMullan PW, et al. Strategies for improving survival after in-hospital cardiac arrest in the United States: 2013 consensus recommendations: a consensus statement from the American Heart Association. Circulation 2013; 127(14):1538-63.

9. Kelsey SF, Abramson NS, Detre KM, Monroe J, Reinmuth O, Safar P, et al. Randomized clinical study of cardiopulmonary-cerebral resuscitation: Design, methods and patient characteristics. Am J Emerg Med 1986; 4:72-86.

10. Ehlenbach WJ, Barnato AE, Curtis JR, Kreuter W, Koepsell TD, Deyo RA should examine the strategy of initiating open cardiac massage by trained individuals if closed-chest CPR for $\approx 15$ minutes (or less) fails to resuscitate victims in-hospital settings. Prolonged CPR can result in high-quality survival if the patient has a potentially reversible cause for cardiac arrest. In all durations of CPR, IHCA, especially cardiac surgery patients have more odds for survival and favorable neurologic outcomes than OHCA.

et al. Epidemiologic study of in-hospital cardiopulmonary resuscitation in the elderly. N Engl J Med 2009; 361:22-31.

11. Yanagidani T, Kometani $Y$, Tsuli M,Nonoue $T$, Kosaka $Y$. Cardiac arrest during anesthesia-a case of effective open chest massage. Masui. 1991; 40(3):468-71.

12. Twomey D, Das M, Subramanian H, Dunning J. Is internal massage superior to external massage for patients suffering a cardiac arrest after cardiac surgery? Interact CardioVasc Thorac Surg 2008;7(1):151 157.

13. Sanders AB, Kern KB, Ewy GA. Time limitations for open-chest cardiopulmonary resuscitation from cardiac arrest. Crit Care Med 1985;13: 897-898.

14. Kern KB, Sanders AB, Badylak SF, Janas W, Carter AB, Tacker WA, et al. Long-term survival with open-chest cardiac massage after ineffective closed-chest compression in a canine preparation. Circulation 1987;75: 498-503.

15. Fialka C, Sebök C, Kemetzhofer P, Oskar K, Fritz S, Vilmos V. Open-chest cardiopulmonary resuscitation after cardiac arrest in cases of blunt chest or abdominal trauma: a consecutive series of 38 cases. J Trauma. 2004 Oct; 57(4):809-14.

16. Lim GB. Cardiac resuscitation: Potential success of prolonged paediatric CPR. Nat Rev Cardiol. 2013 Apr; 10(4):182.

17. Morrison LJ, Kierzek G, Diekema DS, Sayre MR, Silvers SM, Idris AH, et al. Part 3: ethics: 2010 American Heart Association guidelines for cardiopulmonary resuscitation and emergency cardiovascular care. Circulation. 2010 Nov; 122 (18): S665-S675.

18. Goldberger ZD, Chan PS, Berg RA, Kronick SL, Cooke CR, Lu M et al. Duration of resuscitation efforts and survival after in-hospital cardiac arrest: an observational study. The Lancet 2012. Volume 380, No.9852, 1473-1481 